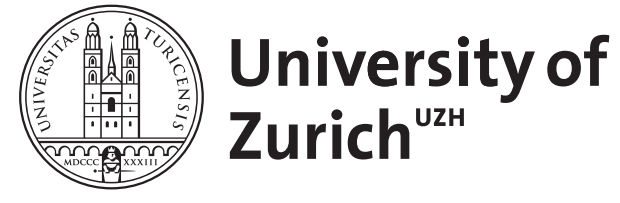

\title{
On the nodal line of the second eigenfunction of elliptic operators in two dimensions
}

Kappeler, T ; Ruf, B

DOI: https://doi.org/10.1515/crll.1989.396.1

Posted at the Zurich Open Repository and Archive, University of Zurich ZORA URL: https://doi.org/10.5167/uzh-22928

Journal Article

Published Version

Originally published at:

Kappeler, T; Ruf, B (1989). On the nodal line of the second eigenfunction of elliptic operators in two dimensions. Journal für die Reine und Angewandte Mathematik, 396:1-13.

DOI: https://doi.org/10.1515/crll.1989.396.1 


\title{
On the nodal line of the second eigenfunction of elliptic operators in two dimensions
}

\author{
By Thomas Kappeler at Philadelphia and Bernhard Ruf at Milano
}

\section{Introduction}

It is a well-known fact that the $n$-th eigenfunction of the Sturm-Liouville eigenvalue equation $-u^{\prime \prime}(x)+q(x) u(x)=\lambda u(x), x \in(0,1), u(0)=u(1)=0$, has exactly $n-1$ nodes (i.e. non-degenerate zeroes), see e.g. [CH].

For the corresponding equation in higher dimensions it is much more complicated to obtain general statements on the zero sets of the eigenfunctions. Let us concentrate on the equation in two dimensions: Let $\Omega$ be a bounded and smooth domain in $\mathbb{R}^{2}$ and $q$ a potential in $C^{\infty}(\Omega)$, and consider

$$
\begin{aligned}
& -\Delta u+q u=\lambda u, \quad \text { in } \Omega \\
& u=0, \quad \text { on } \quad \partial \Omega \text {. }
\end{aligned}
$$

In analogy to the one-dimensional case let $k(n)$ denote the number of connected components of $\Omega \backslash Z_{n}$, where $Z_{n}$ is the nodal set of the $n$-th eigenfunction $u_{n}$ of (1), i.e.

$$
Z_{n}=\left\{x \in \Omega ; u_{n}(x)=0\right\} .
$$

We recall some of the known results concerning equation (1). In [C] one finds the Courant nodal domain theorem, which states that $k(n) \leqq n$ (cf. also [CH]). Pleijel [P] has proved in addition that

$$
\lim _{n \rightarrow \infty} \sup \frac{k(n)}{n} \leqq \frac{4}{j^{2}}<0.7
$$

where $j$ denotes the smallest zero of the 0-th Bessel function. From (2) one concludes that $k(n)=n$ can occur only a finite number of times. There are even examples [S] where the case $k(n)=2$ arises infinitely many times.

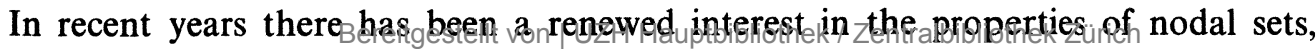
see e.g. $[\mathrm{BG}],[\mathrm{B}],[\mathrm{Be}],[\mathrm{DF}],[\mathrm{HS}],[\mathrm{Y}]$. 
In $[\mathrm{BG}]$ Brüning and Gromes treat the equation

$$
\begin{array}{cccc}
-\Delta u=\lambda u & \text { in } & & \Omega \\
u=0 & \text { on } & \partial \Omega,
\end{array}
$$

where $\Omega$ is a bounded domain of genus $s+1$ with area $F$ and circumference $U$. They prove the following lower bound for the length $l(n)$ of the nodal set of the $n$-th eigenfunction

$$
l(n)+\frac{U}{2} \geqq \frac{F \sqrt{\lambda_{n}}}{2 j}-\pi \frac{j}{2 \sqrt{\lambda_{n}}}(s-1),
$$

where $\lambda_{n}$ denotes the $n$-th eigenvalue and $j$ is the smallest zero of the first Bessel function. With Weyl's asymptotic formula $\lambda_{n} \sim \frac{4 \pi n}{F}$ they then get the following asymptotic lower bound for the length of the nodal line of the $n$-th eigenfunction

$$
\lim _{n \rightarrow \infty} \frac{l(n)+\frac{U}{2}}{F \cdot n} \geqq \frac{\pi}{j^{2}} .
$$

Brüning [B] has generalized this result to compact Riemannian manifolds of dimension 2.

In this paper we show that for equation (1) there exists no upper bound for the length of the nodal line of the second eigenfunction independently of the potential. We prove

Theorem 1. Let $\Omega$ satisfy an interior sphere condition at each point $x \in \partial \Omega$, and let $\Gamma$ be a Lipschitz continuous curve in $\bar{\Omega}$ which divides $\Omega$ into exactly two components. Then there exists for every given $\varepsilon>0$ a potential $q_{\varepsilon}$ in $L^{\infty}(\Omega)$ such that

$$
Z_{2}\left(q_{\varepsilon}\right) \subset[\Gamma]_{\varepsilon}
$$

where $Z_{2}\left(q_{\varepsilon}\right)$ denotes the nodal line of the second eigenfunction of (1) with $q=q_{\varepsilon}$, and $[\Gamma]_{\varepsilon}$ is defined by $[\Gamma]_{\varepsilon}=\{x \in \Omega ; \operatorname{dist}(x, \Gamma) \leqq \varepsilon\}$.

Note that for any given number $L>0$ one can choose a curve $\Gamma$ of length larger than $L+1$ satisfying the conditions of Theorem 1. By Theorem 1 it follows that for $\varepsilon>0$ sufficiently small the length of $Z_{2}\left(q_{\varepsilon}\right)$ is larger than $L$. Hence we have the following

Corollary. There exists no upper bound to the length of the nodal line of the second eigenfunction uniformly for all potentials.

In section 3 we extend the theorem in two directions: First, we give a corresponding result concerning the first $N$ eigenfunctions, and second, we generalize the statements to general second order, symmetric and uniformly operators. 
We mentioned in the beginning that in one dimension the $n$-th eigenfunction $u_{n}$ can be characterized by its $n-1$ nodal points. On the other hand, the $n$-th eigenvalue has also a variational characterization, namely

$$
\lambda_{n}=\min _{F_{n}} \max _{0 \neq v \in E_{n}} \frac{\int\left|v^{\prime}\right|^{2} d x}{\int|v|^{2} d x}
$$

where $F_{n}=\left\{E_{n}=n\right.$-dimensional subspace of $\left.H_{0}^{1}((0,1))\right\}$. Hence, there is a correspondence between the topological characterization (4) of $\lambda_{n}$ and the geometric characterization of $u_{n}$ by the number of nodal points. Many important consequences arise from this relation.

For the problem in higher dimensions one has also a variational characterization of the eigenvalues, but no geometric characterization of the eigenfunctions is known. Our result indicates that such a characterization (if it exists) cannot depend solely on the length of the nodal set.

To prove Theorem 1 we construct explicitely the potential $q_{\varepsilon}$. The idea of the construction is to study carefully an approximation of the variational problem for the Dirichlet integral on the space $H_{\Gamma}=\left\{u \in H_{0}^{1}(\Omega) ; u_{\mid \Gamma}=0\right\}$. This approximation relies on the heuristic observation that adding a potential which is large near $\Gamma$ forces the minimizer to be small near $\Gamma$.

\section{Proof of Theorem 1}

We first introduce some notation. Let us denote by $\Omega$ a bounded domain in $\mathbb{R}^{2}$ with a $C^{1}$ boundary $\partial \Omega$ and with an interior sphere condition at each point $x$ of $\partial \Omega$. Let $\Gamma:[0,1] \rightarrow \bar{\Omega}$ be a Lipschitz continuous (and possibly closed) curve such that $\Omega \backslash \Gamma=\Omega_{1} \cup \Omega_{2}$ consists of exactly two components. Furthermore, let

$$
\Omega_{3 n}=\left\{x \in \Omega_{1} ; \operatorname{dist}(x, \Gamma)<\frac{1}{n}\right\}
$$

and set $\Omega_{1 n}=\Omega_{1} \backslash \bar{\Omega}_{3 n}$. With $\mu_{n 1}<\mu_{n 2} \leqq \cdots$ we denote the Dirichlet eigenvalues corresponding to $\Omega_{1 n}$, i.e. the eigenvalues of $-\Delta y=\lambda y$ on $\Omega_{1 n}$ with $\left.y\right|_{\partial \Omega_{1 n}}=0$. Let $v_{n 1}, v_{n 2}, \ldots$, with $v_{n 1}>0$ on $\Omega_{1 n}$, denote a corresponding orthonormal set of eigenfunctions. Moreover, let $\mu_{1}<\mu_{2} \leqq \cdots$ and $v_{1}<v_{2} \leqq v_{3} \leqq \cdots$ denote the Dirichlet eigenvalues of $\Omega_{1}$ and $\Omega_{2}$, respectively, with corresponding sets of eigenfunctions $v_{1}, v_{2}, \ldots$, and $w_{1}, w_{2}, \ldots$. By the monotone dependence of the eigenvalues on the domain (see Courant-Hilbert $[\mathrm{CH}])$ the sequences $\left(\mu_{n k}\right)_{n \in \mathbb{N}}, k \in \mathbb{N}$, are monotone increasing and

$$
\lim _{n \rightarrow \infty} \mu_{n k}=\mu_{k}, k \in \mathbb{N}
$$

Let us assume without loss of generality that $\mu_{1}<v_{1}$ (if $\mu_{1}>v_{1}$ interchange $\Omega_{1}$ and

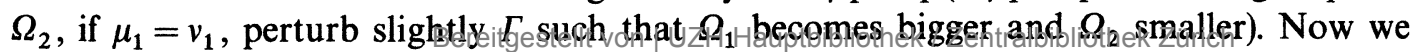
choose a positive convergent sequence $\left(a_{n}\right)_{n \in \mathbb{N}}$ such that for $A n \eta \eta_{n} n^{2} a_{n k}+a_{n}$ and 
$\eta_{k}=\mu_{k}+a$, where $a=\lim _{n \rightarrow \infty} a_{n}$, the following inequalities hold:

and

$$
\eta_{n 1}<v_{1}<\eta_{n 2}, \quad n \in \mathbb{N}
$$

$$
\eta_{1}=v_{1}<\eta_{2}
$$

We now introduce the following sequence $\left(q_{n}\right)_{n \in \mathbb{N}}$ of potentials, i.e. functions defined on $\Omega$, by

$$
q_{n}(x):=r_{n} 1_{\Omega_{3 n}}(x)+a_{n} 1_{\Omega_{1 n}}(x),
$$

where $r_{n}>0$ are real numbers which will be specified later.

We consider the eigenvalue equation

$$
-\Delta y+q_{n} y=\lambda y, y \in H_{0}^{1}(\Omega) \cap H^{2}(\Omega) .
$$

Let $\lambda_{n 1}<\lambda_{n 2} \leqq \lambda_{n 3} \leqq \cdots$ denote the eigenvalues of (5), and $u_{n 1}, u_{n 2}, \ldots$ a corresponding set of $L^{2}(\Omega)$-orthonormal eigenfunctions. With the notations introduced above we get

Lemma 1. The following estimates hold independently of $n \in \mathbb{N}$ :
1) $0 \leqq \lambda_{n 2} \leqq v_{1}$,
2) $\left\|\nabla u_{n 2}\right\|_{L^{2}(\Omega)}^{2} \leqq v_{1}$,
3) $\left\|\Delta u_{n 2}\right\|_{L^{2}\left(\Omega_{2}\right)} \leqq v_{1}$,
4) $\left\|\Delta u_{n 2}\right\|_{L^{2}\left(\Omega_{1 n}\right)} \leqq \sup \left\{v_{1}, a_{n} ; n \in \mathbb{N}\right\}$.

Remark. All the bounds are independent of the sequence $\left(r_{n}\right)_{n \in \mathbb{N}}$.

Proof. By the variational characterization of the second eigenvalue we get

$$
\begin{array}{r}
\lambda_{n 2}=\inf \left\{\|\nabla u\|_{L^{2}(\Omega)}^{2}+r_{n}\|u\|_{L^{2}\left(\Omega_{3 n}\right)}^{2}+a_{n}\|u\|_{L^{2}\left(\Omega_{1 n}\right)}^{2} ;\right. \\
\left.u \in H_{0}^{1}(\Omega),\|u\|_{L^{2}(\Omega)}=1,\left\langle u, u_{n 1}\right\rangle=0\right\},
\end{array}
$$

where $\langle\cdot, \cdot\rangle$ denotes the usual inner product in $L^{2}(\Omega)$. Let us denote by $\bar{v}_{n 1}$ and $\bar{w}_{1}$ the functions $v_{n 1}$ and $w_{1}$, respectively, extended by zero to the whole of $\Omega$. Choose $\delta_{n 1}$ and $\delta_{n 2}$ with $\delta_{n 1}^{2}+\delta_{n 2}^{2}=1$ and $\left\langle\delta_{n 1} \bar{v}_{n 1}+\delta_{n 2} \bar{w}_{1}, u_{n 1}\right\rangle=0$. As $y_{n}:=\delta_{n 1} v_{n 1}+\delta_{n 2} \bar{w}_{1}$ is in $H_{0}^{1}(\Omega)$ and $\left\|y_{n}\right\|_{L^{2}(\Omega)}=1$ one gets from the variational characterization of $\lambda_{n 2}$

$$
\begin{aligned}
\lambda_{n 2} & \leqq \delta_{n 1}^{2}\left(\left\|\nabla v_{n 1}\right\|_{L^{2}\left(\Omega_{1 n}\right)}^{2}+a_{n}\left\|v_{n 1}\right\|_{L^{2}\left(\Omega_{1 n}\right)}^{2}\right)+\delta_{n 2}^{2}\left\|\nabla w_{1}\right\|_{L^{2}\left(\Omega_{2}\right)}^{2} \\
& \leqq \delta_{n 1}^{2} \eta_{n 1}+\delta_{n 2}^{2} v_{1} \leqq v_{1} .
\end{aligned}
$$

So 1) and 2) follow. 4) follows from the fact that $u_{n 2}$ satisfies $-\Delta u_{n 2}=\left(\lambda_{n 2}-a_{n}\right) u_{n 2}$ on

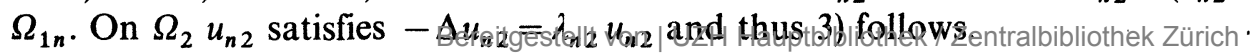


Using lemma 1 we may and do assume for the rest of this paper that the sequence $\left(q_{n}\right)_{n \in \mathbb{N}}$ has been chosen in such a way that

(a) $\left(\lambda_{n 2}\right)_{n \in \mathbb{N}}$ converges; the limit is denoted by $\lambda_{2}$;

(b) $\left(u_{n 2}\right)_{n \in \mathbb{N}}$ converges weakly in $H_{0}^{1}(\Omega)$; the limit function in $H_{0}^{1}(\Omega)$ is denoted by $u_{2}$

c) $\lim _{n \rightarrow \infty} u_{n 2}=u_{2}$ weakly in $H^{2}\left(\Omega_{2}\right)$ and weakly in $H^{2}\left(\Omega_{1 m}\right)$ for $m=1,2, \ldots$

In the following lemma we show that the limit function $u_{2}$ can be identified if we choose suitably the sequence $\left(r_{n}\right)_{n \in \mathbb{N}}$.

Denote by $\bar{v}_{1}$ and $\bar{w}_{1}$ the functions $v_{1}$ and $w_{1}$, respectively, extended by zero to the whole of $\Omega$. Let $d_{n}$ be the interpolation constant (see [LM]) of the following type

$$
\|u\|_{H^{1 / 2}\left(\Omega_{3 n}\right)} \leqq d_{n}\|u\|_{L^{2}\left(\Omega_{3 n}\right)}^{1 / 2} \cdot\|u\|_{H^{1}\left(\Omega_{3 n}\right)}^{1 / 2}\left(u \in H^{1}\left(\Omega_{3 n}\right)\right) .
$$

Moreover, let $c_{n}$ be the embedding constant for

$$
H^{1 / 2}\left(\Omega_{3 n}\right) \hookrightarrow L^{2}\left(\partial \Omega_{3 n}\right) .
$$

Lemma 2. If $\left(r_{n}\right)_{n \in \mathbb{N}}$ satisfies $\lim _{n \rightarrow \infty} \frac{c_{n} d_{n}}{r_{n}^{1 / 4}}=0$, then there exist constants $\alpha$ and $\beta$ with $\alpha^{2}+\beta^{2}=1$ such that

1) $\lambda_{2}=v_{1}\left(=\eta_{1}\right)$,

2) $u_{2}=\alpha \bar{v}_{1}+\beta \bar{w}_{1}$,

3) $\lim _{n \rightarrow \infty} u_{n 2}=\alpha v_{1}$ strongly in $H^{k}\left(\Omega_{1 m}\right)($ all $m$ and $k$ ),

4) $\lim _{n \rightarrow \infty} u_{n 2}=\beta w_{1}$ strongly in $H^{k}\left(\Omega_{2}\right)($ all $k)$.

Proof. As $H_{0}^{1}(\Omega)$ is compactly imbedded in $L^{2}(\Omega)$ one concludes that $\lim _{n \rightarrow \infty} u_{n 2}=u_{2}$ strongly in $L^{2}(\Omega)$. On $\Omega_{2}$ we have $-\Delta u_{n 2}=\lambda_{n 2} u_{n 2}$ and thus $-\Delta u_{2}=\lambda_{2} u_{2}$ and the convergence holds strongly in $H^{2}\left(\Omega_{2}\right)$. Similarly one argues for $\Omega_{1 m}$. So 1), 3) and 4) follow immediately from 2 ).

To prove 2) we first show that $\left.u_{2}\right|_{\Omega_{2}} \in H_{0}^{1}\left(\Omega_{2}\right)$. Since $\lim _{n \rightarrow \infty} u_{n 2}=u_{2}$ weakly in $H^{1}\left(\Omega_{2}\right)$ we conclude by the trace embedding theorem (see [LM]) that $u_{2}=\lim _{n \rightarrow \infty} u_{n 2}$ weakly in $H^{1 / 2}\left(\partial \Omega_{2}\right)$; but $H^{1 / 2}\left(\partial \Omega_{2}\right) \hookrightarrow L^{2}\left(\partial \Omega_{2}\right)$ compactly, and thus $u_{2}=\lim _{n \rightarrow \infty} u_{n 2}$ strongly in $L^{2}\left(\partial \Omega_{2}\right)$. Since $\Omega \cap \partial \Omega_{2} \subset \partial \Omega_{3 n}$ we get the following estimate:

$$
\begin{aligned}
& \left\|\left.u_{n 2}\right|_{\Omega \cap \partial \Omega_{2}}\right\|_{L^{2}\left(\Omega \cap \partial \Omega_{2}\right)} \leqq\left\|\left.u_{n 2}\right|_{\partial \Omega_{3 n}}\right\|_{L^{2}\left(\partial \Omega_{3 n}\right)} \\
& \leqq c_{n}\left\|u_{n 2}\right\|_{H^{1 / 2}\left(\Omega_{3 n}\right)}
\end{aligned}
$$

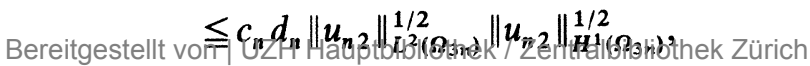


where $c_{n}$ and $d_{n}$ are the imbedding and interpolation constants defined above. The two norms in the last term can be estimated as follows: By lemma 1 we know that

$$
\left\|u_{n 2}\right\|_{H^{1}\left(\Omega_{3 n}\right)}^{2} \leqq\left\|u_{n 2}\right\|_{H_{0}^{1}(\Omega)}^{2} \leqq v_{1}+1 .
$$

Using

$$
\int_{\Omega}\left|\nabla u_{n 2}\right|^{2} d x+a_{n} \int_{\Omega_{1 n}} u_{n 2}^{2} d x+r_{n} \int_{\Omega_{3 n}} u_{n 2}^{2} d x=\lambda_{n 2} \int_{\Omega} u_{n 2}^{2} d x \leqq v_{1}
$$

one concludes that

$$
\left\|u_{n 2}\right\|_{L^{2}\left(\Omega_{3 n}\right)}^{2}=\int_{\Omega_{3 n}} u_{n 2}^{2} d x \leqq \frac{v_{1}}{r_{n}}
$$

Thus we obtain

$$
\left\|\left.u_{n 2}\right|_{\Omega \cap \partial \Omega_{2}}\right\|_{L^{2}\left(\Omega \cap \partial \Omega_{2}\right)} \leqq c_{n} d_{n}\left(\frac{v_{1}}{r_{n}}\right)^{1 / 4}\left(1+v_{1}\right)^{1 / 4}
$$

By the choice of the sequence $\left(r_{n}\right)_{n \in \mathbb{N}}$ we get

$$
\left.u_{2}\right|_{\Omega \cap \partial \Omega_{2}}=\left.\lim _{n \rightarrow \infty} u_{n 2}\right|_{\Omega \cap \partial \Omega_{2}}=0 \text { strongly in } L^{2}\left(\Omega \cap \partial \Omega_{2}\right) .
$$

But $u_{2}$ is in $H_{0}^{1}(\Omega)$ and thus $u_{2 \mid \partial \Omega_{2}}=0$ in $L^{2}\left(\partial \Omega_{2}\right)$.

Since $\Omega \cap \partial \Omega_{2}=\Omega \cap \partial \Omega_{1}$ it follows that $u_{2}$ is also in $H_{0}^{1}\left(\Omega_{1}\right)$. On $\Omega_{1 m}(m \in \mathbb{N})$ the equation $-\Delta u_{2}=\left(\lambda_{2}-a\right) u_{2}$ holds. As $u_{2}$ is in $H_{0}^{1}(\Omega)$ we conclude that $-\Delta u_{2}=\left(\lambda_{2}-a\right) u_{2}$ holds on $\bigcup_{n \in \mathbb{N}} \Omega_{1 n}=\Omega_{1}$. To summarize: $u_{2} \in H_{0}^{1}(\Omega)$ satisfies

$$
\begin{array}{rlll}
-\Delta u_{2}+a u_{2}=\lambda_{2} u_{2} & \text { on } & \Omega_{1}, & \left.u_{2}\right|_{\Omega_{1}} \in H_{0}^{1}\left(\Omega_{1}\right), \\
-\Delta u_{2}=\lambda_{2} u_{2} & \text { on } & \Omega_{2}, & \left.u_{2}\right|_{\Omega_{2}} \in H_{0}^{1}\left(\Omega_{2}\right) .
\end{array}
$$

From $\left\|u_{n 2}\right\|_{L^{2}(\Omega)}=1, n \in \mathbb{N}$, we conclude that $\left\|u_{2}\right\|_{L^{2}(\Omega)}=1$ and hence $u_{2} \neq 0$. Then $\left.u_{2}\right|_{\Omega_{1}} \neq 0$ or $\left.u_{2}\right|_{\Omega_{2}} \neq 0$. Let us assume that $\left.u_{2}\right|_{\Omega_{2}} \neq 0$. (The case $\left.u_{2}\right|_{\Omega_{1}} \neq 0$ is treated similarly.) Due to the fact that $\lambda_{2} \leqq v_{1}$ and $v_{1}$ is the lowest Dirichlet eigenvalue on $\Omega_{2}$ we conclude that $\lambda_{2}=v_{1}$. Together with $\eta_{1}=v_{1}$ this implies that $u_{2}=\alpha \bar{v}_{1}+\beta \bar{w}_{1}$ with $\alpha^{2}+\beta^{2}=1$.

Let us now look at the nodal lines of $u_{n 2}$. We assume that the constants $\alpha$ and $\beta$ in $u_{2}=\alpha \bar{v}_{1}+\beta \bar{w}_{1}$ satisfy $\alpha \geqq 0$ and $\beta \leqq 0$. The case $\alpha \leqq 0$ and $\beta \geqq 0$ is treated similarly. We first consider the case where $\alpha>0$ and $\beta<0$. With this assumption we prove in the following lemma that the nodal line $Z_{n}$ of $u_{n 2}$ approaches $\Gamma$ (except possibly near the boundary $\partial \Omega$ ).

Lemma 3. Assume that $u_{2}=\alpha \bar{v}_{1}+\beta \bar{w}_{1}$ with $\alpha>0$ and $\beta<0$. Let $U, V$ be arbitrary open sets with $\bar{U} \subset \Omega_{1}$ and $\bar{V} \subset \Omega_{2}$. Then there exists a number $n_{0}$ such that $\bar{U} \cap Z_{n}=\emptyset$

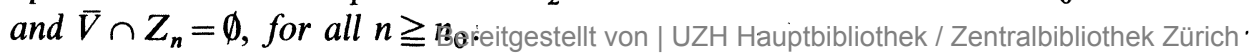


Proof. From lemma 2 we know that $\lim _{n \rightarrow \infty} u_{n 2}=u_{2}$ strongly in $H^{2}(U)$. But $H^{2}(U) \hookrightarrow C(\bar{U})$ is a compact embedding and thus $\lim _{n \rightarrow \infty} u_{n 2}=u_{2}$ in $C(\bar{U})$. By the strict positivity of the first eigenfunctions $v_{1}, w_{1}$ on $\Omega_{1}, \Omega_{2}$, respectively, we get

$$
\min \left\{u_{2}(x) ; x \in \bar{U}\right\}=\min \left\{\alpha v_{1}(x) ; x \in \bar{U}\right\} \geqq \varepsilon>0,
$$

and hence there exists an $n_{0}(\alpha)$ such that

$$
u_{n 2}(x) \geqq \frac{\varepsilon}{2}, \text { for all } n \geqq n_{0}(\alpha), x \in \bar{U} .
$$

This means that $\bar{U} \cap Z_{n}=\emptyset$ for all $n \geqq n_{0}(\alpha)$. Similarly one can argue for $V$.

If $\Gamma$ is a closed curve lying in $\Omega$, then we can easily complete the proof of the theorem. In fact, in this case the whole nodal line $Z_{n}$ of $u_{n 2}$ lies either near the boundary $\partial \Omega$ or near $\Gamma$ (for $n$ sufficiently large). The first possibility cannot occur. To see it, denote by $D_{n}$ the domain lying between $Z_{n}$ and $\partial \Omega$. Then $-\Delta u_{n 2}=\lambda_{n 2} u_{n 2}$ in $D_{n}$ with $u_{n 2}=0$ on $\partial D_{n}$ (if $D_{n} \subset \Omega_{1}$ a term $a_{n} u_{n 2}$ has to be added on the left). Assuming that $Z_{n}$ lies near the boundary $\partial \Omega$ the area $\left|D_{n}\right|$ gets arbitrarily small, contradicting that $\lambda_{n 2} \leqq v_{1}$ for all $n \in \mathbb{N}$. We remark that in this case no interior sphere condition on $\partial \Omega$ is needed.

If the curve $\Gamma$ meets the boundary $\partial \Omega$ then we need an additional argument to complete the proof of the theorem (still in the case $\alpha>0$ and $\beta<0$ ).

Lemma 4. For every $\varepsilon>0$ there exists a number $n_{0}$ and constants $\delta, \gamma>0$ such that

$$
\left|u_{n 2}(x)\right| \geqq \gamma \cdot \operatorname{dist}\left(x, \partial \Omega \backslash[\Gamma]_{\varepsilon}\right)\left(x \in\left([\partial \Omega]_{\delta} \cap \Omega\right) \backslash[\Gamma]_{\varepsilon}\right) .
$$

Proof. By lemma 2 and by using Sobolev's embedding theorem

$$
H^{3}\left(\Omega_{2} \cup \Omega_{1 m}\right) \hookrightarrow C^{1}\left(\overline{\Omega_{2} \cup \Omega_{1 m}}\right),
$$

it follows that

$$
\lim _{n \rightarrow \infty} \sup _{x \in \partial \Omega \backslash[\Gamma]_{\varepsilon}}\left|\partial_{v}\left(u_{n 2}(x)-u_{2}(x)\right)\right|=0,
$$

where $v$ denotes the inside normal to $\partial \Omega$, and $\partial_{v}$ the derivative in the direction $v$. From [GT], p. 33, we conclude, recalling that $\alpha v_{1}>0$ in $\Omega_{1}$ and $\beta w_{1}<0$ in $\Omega_{2}$, and using the interior sphere condition on $\partial \Omega$, that for a given $\varepsilon>0$ there exists a $\gamma>0$ such that

$$
\begin{array}{lll}
\alpha \partial_{v} v_{1}(x) \geqq 2 \gamma, & \text { for all } & x \in \partial \Omega_{1} \backslash[\Gamma]_{\varepsilon}, \\
\beta \partial_{v} w_{1}(x) \leqq-2 \gamma, & \text { for all } & x \in \partial \Omega_{2} \backslash[\Gamma]_{\varepsilon} .
\end{array}
$$

Hence there exists a number $n_{0}$ and a constant $\delta>0$ such that

$$
\left|u_{n 2}(x)\right| \geqq \gamma \cdot \operatorname{dist}\left(x, \partial \Omega \backslash[\Gamma]_{\varepsilon}\right)
$$


In the case that $u_{2}=\alpha \bar{v}_{1}+\beta \bar{w}_{1}$ with $\alpha>0$ and $\beta<0$ this completes the proof of the theorem, since by lemma 3 and 4 there exists a number $k_{0}$ such that $u_{k 2} \neq 0$ for all $x \in \Omega \backslash[\Gamma]_{\varepsilon}$, for all $k \geqq k_{0}$.

In the case that $\alpha=0$ or $\beta=0$ we need some additional results to investigate the nodal lines. We consider here the case that $\alpha=0$. The case $\beta=0$ is treated similarly.

Let $u_{n 2}^{+}:=\max \left\{u_{n 2}, 0\right\}$, and $f_{n}:=u_{n 2}^{+} /\left\|u_{n 2}^{+}\right\|_{L^{2}(\Omega)}$. Due to $\left\langle u_{n 1}, u_{n 2}\right\rangle=0$ we have $\left\|u_{n 2}^{+}\right\|_{L^{2}(\Omega)} \neq 0$ for all $n \in \mathbb{N}$ and thus $f_{n}$ is well defined.

Lemma 5. $\lim _{n \rightarrow \infty} f_{n}=\bar{v}_{1}$ weakly in $H_{0}^{1}(\Omega)$ and a.e. in $\Omega$.

Proof. We have $f_{n} \in H_{0}^{1}(\Omega)$, for all $n \in \mathbb{N}$ (see [LM]). From

$$
-\Delta u_{n 2}+q_{n} u_{n 2}=\lambda_{n 2} u_{n 2}
$$

we get by scalar multiplication with $f_{n}$ and division by $\left\|u_{n 2}^{+}\right\|$

$$
\int_{\Omega}\left|\nabla f_{n}\right|^{2} d x+\int_{\Omega} q_{n} f_{n}^{2} d x=\lambda_{n 2} \int_{\Omega} f_{n}^{2} d x \leqq v_{1}
$$

and hence $\left\|f_{n}\right\|_{H_{0}^{1}(\Omega)}^{2} \leqq v_{1}+1$.

To prove the lemma it is sufficient to show that for every subsequence of $\left(f_{n}\right)_{n \in \mathbb{N}}$ there exists another one which converges weakly to $\bar{v}_{1}$ in $H_{0}^{1}(\Omega)$. As $\left(f_{n}\right)_{n \in \mathbb{N}}$ is bounded in $H_{0}^{1}(\Omega)$ there exists a subsequence of $\left(f_{n}\right)_{n \in \mathbb{N}}$, again denoted by $\left(f_{n}\right)_{n \in \mathbb{N}}$, and a function $f$ in $H_{0}^{1}(\Omega)$ such that $f=\lim _{n \rightarrow \infty} f_{n}$ weakly in $H_{0}^{1}(\Omega)$.

According to lemma $\left.3 f\right|_{\bar{V}}=0$ for every open set $V$ with $\bar{V} \subset \Omega_{2}$. Thus $\left.f\right|_{\Omega_{2}}=0$. Since $\left.f\right|_{\Omega_{2}} \in H^{1}\left(\Omega_{2}\right)$ we conclude by the trace embedding theorem that $\left.f\right|_{\partial \Omega_{2}}=0$ in $L^{2}\left(\partial \Omega_{2}\right)$. Since $f \in H_{0}^{1}(\Omega)$ this implies that $\left.f\right|_{\partial \Omega_{1}}=0$ in $L^{2}\left(\partial \Omega_{1}\right)$, also. This shows that $f$ is in $H_{0}^{1}\left(\Omega_{1}\right)$.

From (7) we get

$$
\int_{\Omega}\left|\nabla f_{n}\right|^{2} d x<\lambda_{n 2}-a_{n} \int_{\Omega_{1 n}} f_{n}^{2} d x
$$

Now $\Omega_{1 n} \subset \Omega_{1(n+1)} \subset \cdots$ with $\bigcup_{n \in \mathbb{N}} \Omega_{1 n}=\Omega_{1}$, and $f=\lim _{n \rightarrow \infty} f_{n}$ strongly in $L^{2}(\Omega)$, thus $\lim _{n \rightarrow \infty} \int_{\Omega_{1 n}} f_{n}^{2} d x=\int_{\Omega_{1}} f^{2} d x=1$, where the last equality follows from $\int_{\Omega} f^{2} d x=1$ and $\left.f\right|_{\Omega_{2}}=0$. Using $\lim _{n \rightarrow \infty} \lambda_{n 2}=v_{1}=\eta_{1}$ and $\lim _{n \rightarrow \infty} a_{n}=a$ we obtain

$$
\int_{\Omega_{1}}|\nabla f|^{2} d x \leqq \int_{\Omega}|\nabla f|^{2} d x \leqq \limsup _{n \rightarrow \infty} \int_{\Omega}\left|\nabla f_{n}\right|^{2} d x \leqq \eta_{1}-a=\mu_{1} .
$$

Since $\mu_{1}$ is the lowest Dirichlet eigenvalue of $\Omega_{1}$ and $f \in H_{0}^{1}\left(\Omega_{1}\right)$ with $\|f\|_{L^{2}\left(\Omega_{1}\right)}=1$ and $f>0$ we conclude that $\int_{\Omega_{1}}|\nabla f|^{2} d x=\mu_{1}$ and $f=\bar{v}_{1}$. 
Let us introduce the sets $F_{k}=\left\{x \in \Omega ; u_{k 2}(x)>0\right\}$ and

$$
B_{n}:=\bigcap_{k \geqq n} F_{k}=\left\{x \in \Omega ; u_{k 2}(x)>0 \text { for all } k \geqq n\right\}
$$

We get the following

Corollary 6. $\Omega_{1} \backslash \bigcup_{n \in \mathbb{N}} B_{n}$ is a null set.

Proof. $x$ is an element of $\Omega_{1} \backslash \bigcup_{n \in \mathbb{N}} B_{n}$ iff $x$ is in $\Omega_{1}$ and $x \notin \Omega_{1} \cap B_{n}$ for all $n \in \mathbb{N}$, that is for each $n \in \mathbb{N}$ there exists a $k(n) \geqq n$ such that $x \notin \Omega_{1} \cap F_{k(n)}$, i.e. $u_{k(n) 2}^{+}=0$, $n \in \mathbb{N}$. But then $f_{k(n)}(x)=0(n \in \mathbb{N})$ and thus $\lim _{n \rightarrow \infty} f_{k(n)}(x)=0$. As $\lim _{n \rightarrow \infty} f_{n}=\bar{v}_{1}$ a.e. and $v_{1}>0$ in $\Omega_{1}$ the claim follows.

Let us now fix $m$ and introduce the functions

$$
g_{m n}=u_{n 2} 1_{\Omega_{1 m} \cup F_{n}}, \quad h_{m n}=g_{m n} /\left\|g_{m n}\right\|_{L^{2}(\Omega)}
$$

Observe that $\left\|g_{m n}\right\|_{L^{2}(\Omega)} \neq 0$ and hence $h_{m n}$ is well-defined.

Lemma 7. Fix $m \geqq 1$. Then

1) $\lim _{n \rightarrow \infty} h_{m n}^{+}=c \bar{v}_{1} \quad$ weakly in $H_{0}^{1}(\Omega)$ and a.e. with $0 \leqq c \leqq 1$,

2) $\lim _{n \rightarrow \infty} h_{m n}=\bar{v}_{1} \quad$ strongly in $L^{2}(\Omega)$,

3) $\lim _{n \rightarrow \infty} h_{m n}=v_{1} \quad$ strongly in $H^{k}\left(\Omega_{1 m}\right), \quad k=1,2, \ldots$

Proof. 1) is proved in the same way as lemma 5. Observe that now $\left\|h_{m n}^{+}\right\|_{L^{2}(\Omega)} \leqq 1$ so we can only conclude that $0 \leqq c \leqq 1$. 2) From the definition of $h_{m n}$ one sees that $h_{m n} 1_{\Omega \backslash \Omega_{1 m}}=h_{m n}^{+} 1_{\Omega \backslash \Omega_{1 m}}$ and that $\lim _{n \rightarrow \infty} h_{m n} 1_{\Omega \backslash \Omega_{1 m}}=c \bar{v}_{1} 1_{\Omega \backslash \Omega_{1 m}}$ strongly in $L^{2}(\Omega)$. On the other hand $\left(h_{m n} 1_{\Omega_{1 m}}\right)_{n \geqq m}$ is bounded in $H^{2}\left(\Omega_{1 m}\right)$. Thus there is a subsequence of $\left(h_{m n} 1_{\Omega_{1 m}}\right)_{n \geqq m}$ (denoted the same) such that $\lim _{n \rightarrow \infty} h_{m n} 1_{\Omega_{1 m}}$ exists strongly in $L^{2}\left(\Omega_{1 m}\right)$. This proves that there exists $h \in L^{2}(\Omega),\|h\|_{L^{2}(\Omega)}=1$, with $\lim _{n \rightarrow \infty} h_{m n}=h$ strongly in $L^{2}(\Omega)$. In consequence $\lim _{n \rightarrow \infty} h_{m n}^{+}=h^{+}=c \bar{v}_{1}$ and $\lim _{n \rightarrow \infty} h_{m n}^{-}=h^{-}$strongly in $L^{2}(\Omega)$. Note that $\operatorname{supp} \bar{v}_{1}=\bar{\Omega}_{1}$ and supp $h^{-} \subset \bar{\Omega}_{1 m} \subset \bar{\Omega}_{1}$. Thus only $c=1$ or $c=0$ are possible. But $c=0$ is impossible because in this case $\left\|h^{-}\right\|_{L^{2}(\Omega)}=1$, and this would contradict Corollary 6 . So 2) and thus 3) follow.

To complete the proof of the theorem one now applies lemma 3 and 4 to the sequence $\left(h_{m n}\right)_{n \in \mathbb{N}}$ to conclude that for every $\varepsilon>0$ there exists a number $n_{0}$ such that for

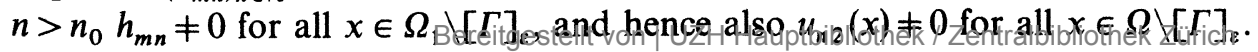




\section{Extensions and generalizations}

I. Here we show that theorem 1 can be generalized to the zero set of the first $N$ eigenfunctions of (1) in the following manner. Let $Z_{j}(q)=\left\{x \in \Omega ; u_{j}(x)=0\right\}$, where $u_{j} \in H_{0}^{1}(\Omega) \cap H^{2}(\Omega)$ solves $-\Delta u_{j}+q u_{j}=\lambda_{j} u_{j}, 2 \leqq j \leqq N$. Assume that there are Lipschitz continuous curves $\gamma_{i}:[0,1] \rightarrow \bar{\Omega}, 1 \leqq i \leqq k$, with $k \leqq N$, such that $\Omega \backslash \Gamma$ has exactly $N$ components, where $\Gamma=\bigcup_{i=1}^{k} \gamma_{i}$. Assume that $\Omega$ satisfies an interior sphere condition
for all $x \in \partial \Omega$.

Theorem 2. There exists for every $\varepsilon>0$ a potential $q_{\varepsilon} \in L^{\infty}(\Omega)$ such that

$$
Z_{j}\left(q_{\varepsilon}\right) \subset[\Gamma]_{\varepsilon}, \quad 2 \leqq j \leqq N .
$$

Proof. The proof follows the same lines as the proof of theorem 1, and we just point out the main differences.

Let $\Omega_{1}, \ldots, \Omega_{N}$ denote the components of $\Omega \backslash \Gamma$, and assume without loss of generality that $\mu_{1}\left(\Omega_{1}\right)<\mu_{1}\left(\Omega_{2}\right)<\cdots<\mu_{1}\left(\Omega_{N}\right)$, where $\mu_{1}(D)$ denote the first eigenvalue of

$$
\begin{aligned}
-\Delta u & =\lambda u, \quad \text { in } \quad D, \\
u & =0, \quad \text { on } \quad \partial D .
\end{aligned}
$$

By $v_{i}:=v_{1}\left(\Omega_{i}\right), i=1, \ldots, N$, we denote the corresponding first eigenfunctions, with $\left\|v_{i}\right\|_{L^{2}\left(\Omega_{i}\right)}=1$, and $v_{i}>0$ in $\Omega_{i}$. We set $[\Gamma]_{1 / n}=\{x \in \Omega$; dist $(\Gamma, x)<1 / n\}$ and let

$$
\Omega_{n i}=\Omega_{i} \backslash[\Gamma]_{1 / n} \quad i=1, \ldots, N .
$$

Set $\mu_{n i}:=\mu_{1}\left(\Omega_{n i}\right), i=1, \ldots, N$, and $v_{n i}$ the corresponding first eigenfunctions (chosen positive and with $\left.\left\|v_{n i}\right\|_{L^{2}\left(\Omega_{n i}\right)}=1\right)$. The sequences $\left(\mu_{n i}\right)_{n \geqq 1}$ are monotonically decreasing and $\mu_{n i} \rightarrow \mu_{1}\left(\Omega_{i}\right)$ for $n \rightarrow \infty(1 \leqq i \leqq N)$. Now choose positive convergent sequences $\left(a_{n i}\right)_{n \in \mathbb{N}}(i=1, \ldots, N-1)$ with $a_{i}=\lim _{n \rightarrow \infty} a_{n i}$, such that for

$$
\eta_{n i}:=\mu_{n i}+a_{n i} \text { and } \eta_{1}\left(\Omega_{i}\right)=\mu_{1}\left(\Omega_{i}\right)+a_{i}
$$

the following inequalities hold (for all $n \in \mathbb{N}$ ):

and

$$
\eta_{n 1}<\eta_{n 2}<\cdots<\eta_{n(N-1)}<\mu_{1}\left(\Omega_{N}\right)
$$

$$
\eta_{1}\left(\Omega_{i}\right)=\mu_{1}\left(\Omega_{N}\right)<\eta_{2}\left(\Omega_{i}\right), \quad i=1, \ldots, N-1
$$

Finally, let $q_{n}(x)=r_{n} 1_{[\Gamma]_{1 / n}}(x)+\sum_{i=1}^{N-1} a_{n i} 1_{\Omega_{n i}}(x)$, where $r_{n}$ are constants which will be chosen later. We denote by $\lambda_{n 1}<\lambda_{n 2} \leqq \lambda_{n 3} \leqq \cdots$ the Dirichlet eigenvalues of

$$
-\Delta y+q_{n} y=\lambda y, \quad \text { in } \Omega, \quad y=0 \text { on } \partial \Omega,
$$

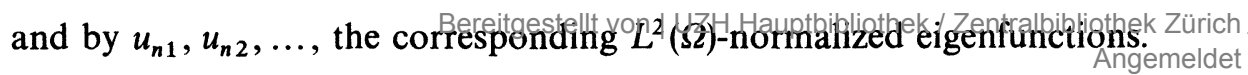


One now proves as in lemma 1 that for all $i \in\{1, \ldots, N\}$ and all $n \in \mathbb{N}$

$$
\begin{gathered}
0 \leqq \lambda_{n i} \leqq \mu_{1}\left(\Omega_{N}\right), \\
\left\|\nabla u_{n i}\right\|_{L^{2}(\Omega)}^{2} \leqq \mu_{1}\left(\Omega_{N}\right), \\
\left\|\Delta u_{n 2}\right\|_{L^{2}\left(\Omega_{n i}\right)} \leqq \sup \left\{\left(\alpha_{i n}, \mu_{1}\left(\Omega_{N}\right)\right\} .\right.
\end{gathered}
$$

In fact, one uses again the variational characterization of the $i$-th eigenvalue $(1 \leqq i \leqq N)$ to obtain

$$
\lambda_{n i} \leqq \sum_{j=1}^{i} \delta_{n j}^{2}\left(\left\|\nabla v_{n j}\right\|_{L^{2}\left(\Omega_{n j}\right)}^{2}+a_{n j}\left\|v_{n j}\right\|_{L^{2}\left(\Omega_{n j}\right)}^{2}\right)
$$

where $a_{n N}:=0(n \in \mathbb{N})$ and $\delta_{n j} \in[-1,1]$ are chosen such that

$$
\sum_{j=1}^{i} \delta_{n j}^{2}=1 \text { and } \sum_{j=1}^{i}\left\langle\delta_{n j} v_{n j}, u_{n k}\right\rangle=0, k=1, \ldots, i-1
$$

One then gets

$$
\lambda_{n i} \leqq \sum_{j=1}^{i} \delta_{n j}^{2} \eta_{n j} \leqq \sum_{j=1}^{i} \delta_{n j}^{2} \mu_{1}\left(\Omega_{N}\right)=\mu_{1}\left(\Omega_{N}\right)
$$

From this (10) and (11) follow, while (12) follows from equation (9) restricted to the sets $\Omega_{n i}$.

As in lemma 2 we denote by $d_{n}$ and $c_{n}$ the interpolation respectively embedding constants for $H^{1 / 2}\left([\Gamma]_{1 / n}\right)$. Choosing $\left(r_{n}\right)_{n \in \mathbb{N}}$ such that $\lim _{n \rightarrow \infty} \frac{c_{n} \cdot d_{n}}{r_{n}^{1 / 4}}=0$ one now proves exactly as in lemma 2 that there exist for all $i \in\{1, \ldots, N\}$ constants $\alpha_{j}:=\alpha_{j}(i)$, $j=1, \ldots, N$, with $\sum_{j=1}^{N} \alpha_{j}^{2}=1$ such that

$$
\begin{gathered}
\lambda_{i}:=\lim _{n \rightarrow \infty} \lambda_{n i}=\mu_{1}\left(\Omega_{N}\right), \\
u_{i}:=\lim _{n \rightarrow \infty} u_{n i}=\sum_{j=1}^{N} \alpha_{j} v_{j} \quad \text { weakly in } H_{0}^{1}(\Omega), \\
\lim _{n \rightarrow \infty} u_{n i}=\alpha_{j} v_{j} \text { strongly in } H^{k}\left(\Omega_{m_{j}}\right), \quad(\text { all } m \text { and } k) .
\end{gathered}
$$

Consider now $u_{i}$ for some fixed $i \in\{2, \ldots, N\}$. Then $\alpha_{j}(i) \neq 0$ for at least one $j \in\{1, \ldots, N\}$. We can assume that $\alpha_{j}>0$. One now shows as in lemma 3 that for an arbitrary open set $U$ with $\bar{U} \subset \Omega_{j}$ there exists a number $n_{0}$ such that $\bar{U} \cap Z_{n j}=\emptyset$, for all $n \geqq n_{0}$, where $Z_{n j}=\left\{x \in \Omega ; u_{n j}(x)=0\right\}$. If $\alpha_{k}=0$ for all $k \in\{1, \ldots, N\}$ then one easily completes the proof as in lemma 4 .

Assume now that $\alpha_{k}=0$ for some $k \in\{1, \ldots, N\}$. Let $\Omega_{m k}=\Omega_{k} \backslash[\Gamma]_{1 / m}$ and let $F_{n}^{+}=\left\{x \in \Omega ; u_{n i}(x)>0\right\}$ and $F_{n}^{-}=\left\{x \in \Omega ; u_{n i}(x)<0\right\}$. The sets $F_{n}^{+}$and $F_{n}^{-}$consist

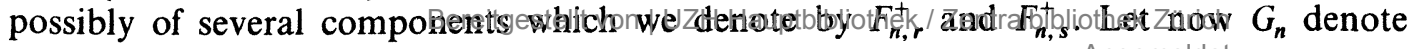


that component of $F_{n}^{+}$or $F_{n}^{-}$which intersects $\Omega_{m k}$, and which satisfies

$$
\left|G_{n} \cap \Omega_{m k}\right| \geqq \max _{r, s}\left\{\left|F_{n, r}^{+} \cap \Omega_{m k}\right|,\left|F_{n, s}^{-} \cap \Omega_{m, k}\right|\right\},
$$

where $|D|$ denotes the Lebesgue-measure of the set $D$. By choosing an appropriate subsequence, if necessary, we may assume that either $G_{n} \subset F_{n}^{+}$or $G_{n} \subset F_{n}^{-}$for all $n \in \mathbb{N}$. Assume that the first is the case.

Let now $\tilde{u}_{n i}:=u_{n i} 1_{G_{n}}$ and $f_{n}:=\tilde{u}_{n i} /\left\|\tilde{u}_{n i}\right\|_{L^{2}(\Omega)}$. As in lemma 5 one now proves that $\lim _{n \rightarrow \infty} f_{n}=\bar{v}_{k}$ weakly in $H_{0}^{1}(\Omega)$ and a.e. in $\Omega$, where $\bar{v}_{k}$ denotes the extension by zero to the whole of $\Omega$ of $v_{k}$ (the first Dirichlet-eigenfunction on $\Omega_{k}$ ).

Also the statement corresponding to lemma 6 follows now easily, namely that $\Omega_{k} \backslash \bigcup_{n \in N} B_{n}$ is a null set, where $B_{n}:=\bigcap_{j \geqq n} G_{j}$.

Finally, one introduces the functions

$$
g_{m n}:=u_{n i} 1_{\Omega_{m k} \cup G_{n}}, \quad h_{m n}:=g_{m n} / g_{m n} \|_{L^{2}(\Omega)}
$$

and proves the statements of lemma 7 , which allows to conclude the proof.

Corollary. For any given $N \in \mathbb{N}$ there exist potentials $q \in L^{\infty}(\Omega)$ such that the length of the nodal line of the first $N$ eigenfunctions of (1) is arbitrarily long.

Proof. Let $L>0$ be given, and let $\gamma_{i}, i=1, \ldots, N-1$, denote disjoint curves of length $l\left(\gamma_{i}\right) \geqq L+1$ and such that $\Omega \backslash \Gamma\left(\right.$ with $\left.\Gamma=\bigcup_{i=1}^{N-1} \gamma_{i}\right)$ has exactly $N$ components. By theorem 2 we can find potentials $q$ such that the nodal sets $Z_{i}, i=2, \ldots, N$, lie in arbitrary $\varepsilon$-neighbourhoods of $\Gamma$. This clearly implies that we can find potentials $q$ such that $l\left(Z_{i}\right)>L$, for $i=2, \ldots, N$, where $l\left(Z_{i}\right):=$ length of $Z_{i}$.

II. Suppose that

$$
A=\sum_{i, j=1}^{2} \frac{\partial}{\partial x_{i}} a_{i j}(x) \frac{\partial}{\partial x_{j}}
$$

is a uniformly elliptic, formally selfadjoint linear differential expression of second order with real-valued coefficient functions $a_{i j}=a_{j i} \in C^{\mathbf{1}}(\bar{\Omega})$, and let $L$ denote the linear operator induced by $A$ in $L^{2}(\Omega)$ with domain $D(L)=H_{0}^{1}(\Omega) \cap H^{2}(\Omega)$. Then $L$ is selfadjoint and has a compact resolvent; the spectrum of $L$ consists of a sequence $\lambda_{1}<\lambda_{2} \leqq \lambda_{3} \leqq \cdots$ of eigenvalues with finite multiplicities.

It is a straightforward application of the general theory of elliptic PDE to prove theorem 1 and 2 for the equation

$$
\begin{aligned}
& A u+q u=\lambda u, \quad \text { in } \Omega \\
& u=0, \quad \text { on } \partial \Omega \text {. }
\end{aligned}
$$

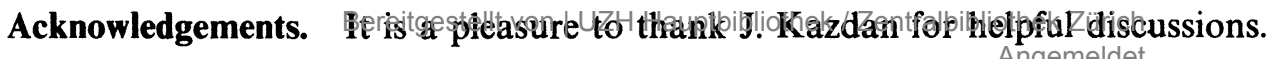




\section{References}

[Be] P. Berard, Volume des ensembles nodaux des fonctions propres du Laplacien, Séminaire BonySjöstrand-Meyer 1984-1985, Exposé No. XIV, 1985, Ecole Polytechnique Palaiseau.

[B] J. Brüning, Über Knoten von Eigenfunktionen des Laplace-Beltrami-Operators, Math. Z. 158 (1978), $15-21$.

[BG] J. Brüning, D. Gromes, Über die Länge der Knotenlinien schwingender Membranen, Math. Z. 124 (1972), 79-82.

[C] S. T. Cheng, Eigenfunctions and nodal sets, Comm. Math. Helv. 51 (1979), 43-55.

[CH] R. Courant, D. Hilbert, Methods of Mathematical Physics, Vol. 1, New York 1962.

[DF] H. Donnelly, C. Fefferman, Nodal sets of eigenfunctions on Riemannian manifolds, preprint.

[GT] D. Gilbarg, N.S. Trudinger, Elliptic Partial Differential Equations of Second Order, Grundl. der Math. Wiss. 224, Berlin-Heidelberg-New York 1977.

[HS] R. Hardt, L. Simon, Nodal sets for solutions of elliptic equations, preprint.

[LM] J. L. Lions, E. Magenes, Non-Homogeneous Boundary Value Problems and Applications, Vol.1, Grundl. der Math. Wiss. 181, Berlin-Heidelberg-New York 1972.

[P] A. Pleijel, Remarks on Courant's nodal domain theorem, Comm. Pure Appl. Math. 9 (1956), 543 - 550.

[S] A. Stern, Bemerkungen über asymptotisches Verhalten von Eigenwerten und Eigenfunktionen, Dissertation, Göttingen 1925.

[Y] S. T. Yau, Problem section, Seminar on differential geometry, Ann. of Math. Stud. 102 (1982), 669706.

Department of Mathematics, University of Pennsylvania, Philadelphia, USA

Università di Milano, Dipartimento di Matematica, Via C. Saldini 50, 20133 Milano, Italia

Eingegangen 5. Juni 1987 